\title{
From orbital to Pauli-limited critical fields in granular aluminum films
}

\author{
Aviv Glezer Moshe $\odot,{ }^{1,{ }^{*}}$ Eli Farber, ${ }^{2}$ and Guy Deutscher ${ }^{1}$ \\ ${ }^{1}$ Raymond and Beverly Sackler School of Physics and Astronomy, Tel Aviv University, Tel Aviv, Israel \\ ${ }^{2}$ Department of Physics and Department of Electrical and Electronic Engineering, Ariel University, Ariel
}

(Received 3 August 2020; revised 2 October 2020; accepted 28 October 2020; published 10 December 2020)

\begin{abstract}
The temperature dependence of the perpendicular upper critical field of superconducting granular aluminum films has been measured for samples approaching the metal-to-insulator transition. Analysis of the results shows a shift from an orbital to a Pauli-limited critical field, which we propose is made possible by electron mass renormalization. In that regime, the critical field transition becomes of the first order, as predicted by Fulde, Ferrel, Larkin, and Ovchinikov. The phase-coherence length $\xi_{\text {phase }}$ and the superconducting gap $\Delta$ obtained from the analysis are consistent with a Bardeen-Cooper-Schrieffer-Bose-Einstein condensate crossover region, which we propose is triggered by the Mott transition of the granular films.
\end{abstract}

DOI: 10.1103/PhysRevResearch.2.043354

\section{INTRODUCTION}

The upper critical field of type-II superconductors is usually limited by orbital effects. It is reached when the cores of Abrikosov vortices, set by the coherence length, overlap. Another mechanism that can quench superconductivity is pair breaking by Pauli spin polarization. It has been observed in films thinner than the coherence length placed in a parallel field, thus avoiding the presence of vortices [1-7]. But in three-dimensional samples, the orbital limit is usually reached well before spin polarization becomes important, in which exceptions are exotic superconductors such as LiFeAs [8] and $\mathrm{LiTi}_{2} \mathrm{O}_{4-\delta}$ [9].

Granular superconductors appear to be another possible exception. At high normal-state resistivity values, their critical field saturates at a value suggesting Pauli limitation [10,11]. Here we study the transition from an orbital to a Pauli-limited critical field and determine their respective values.

Werthamer, Helfand, and Hohenberg (WHH) [12] have developed a theory of the upper critical field that includes both orbital and Pauli limitations. Their relative roles are determined by the value of the Maki parameter,

$$
\alpha=\frac{3 \mu_{B}}{e \tau_{\mathrm{tr}} v_{F}^{2}},
$$

where $\tau_{\text {tr }}$ is the transport scattering time and $v_{F}$ is the Fermi velocity. The transition from orbital to Pauli limitation occurs when alpha is approximately equal to 1 .

By measuring the temperature dependence of the perpendicular upper critical field of granular aluminum films, and fitting it to the WHH theory, we have been able to determine how the Maki parameter varies close to the metal-to-insulator

\footnotetext{
*avivmoshe@mail.tau.ac.il
}

Published by the American Physical Society under the terms of the Creative Commons Attribution 4.0 International license. Further distribution of this work must maintain attribution to the author(s) and the published article's title, journal citation, and DOI. transition. Our key finding is that it increases with resistivity and becomes higher than unity near the transition, which is anomalously high value for a conventional type-II superconductor. The prediction $[13,14]$ of a first-order transition at low temperature in that regime is consistent with our observations.

We show that the large value of the Maki parameter requires a reduced effective Fermi velocity, a key characteristic of a Mott transition [15]. This large value allows one to accurately determine both the values of the orbital limited critical field $H_{c 2}^{\text {orb }}$ and that of the Pauli-limited critical field $H_{P}$. One thus obtains both the value of the phase-coherence length $\xi_{\text {phase }}$ and that of the superconducting gap $\Delta$. We have found that the $\xi_{\text {phase }}$ saturates down to about 6 to $7 \mathrm{~nm}$, while the value of $\Delta$ remains at about $0.5 \mathrm{meV}$. Because at the same time the critical temperature goes down, the strong-coupling ratio $2 \Delta / k_{B} T_{c}$ reaches a value of about 5 . The behaviors of the phase-coherence length and of the strong-coupling ratio are consistent with a BCS-BEC crossover near the Metalinsulator (MI) transition [16].

\section{EXPERIMENTAL AND METHODS}

Granular aluminum thin films were prepared by thermal evaporation of clean $\mathrm{Al}$ pallets in a controlled $\mathrm{O}_{2}$ pressure, where the base pressure of the vacuum chamber is $\sim 1 \times 10^{-7}$ Torr. The films were deposited onto liquid-nitrogen-cooled substrates, either on bare dielectrics $\left[\mathrm{MgO}\right.$ or $\left(\mathrm{LaAlO}_{3}\right)_{0.3}-$ $\left(\mathrm{SrAl}_{0.5} \mathrm{Ta}_{0.5} \mathrm{O}_{3}\right)_{0.7}$ (LSAT)] or onto Hall bars patterned on $\mathrm{Si} / \mathrm{SiO}_{2}$ or $\mathrm{Al}_{2} \mathrm{O}_{3}$ substrates. Films with various degrees of grain coupling were obtained by varying the $\mathrm{O}_{2}$ partial pressure in the range of $2-5 \times 10^{-5}$ Torr, while keeping the deposition rate about $5 \pm 1 \mathrm{~A} / \mathrm{s}$, similar to previous work $[17,18]$. This results in pure $\mathrm{Al}$ grains, $2 \mathrm{~nm}$ in size, embedded in an insulating aluminum oxide $[19,20]$. For more details on the implication for the normal-state properties due to the small grain size, please see [21-23]. The films thickness is in the range of 40-100 $\mathrm{nm}$.

Standard four-point resistivity measurements were performed with either a Keithley 6221 current source and Keithley 2182A nano-voltmeter or with a Stanford research 


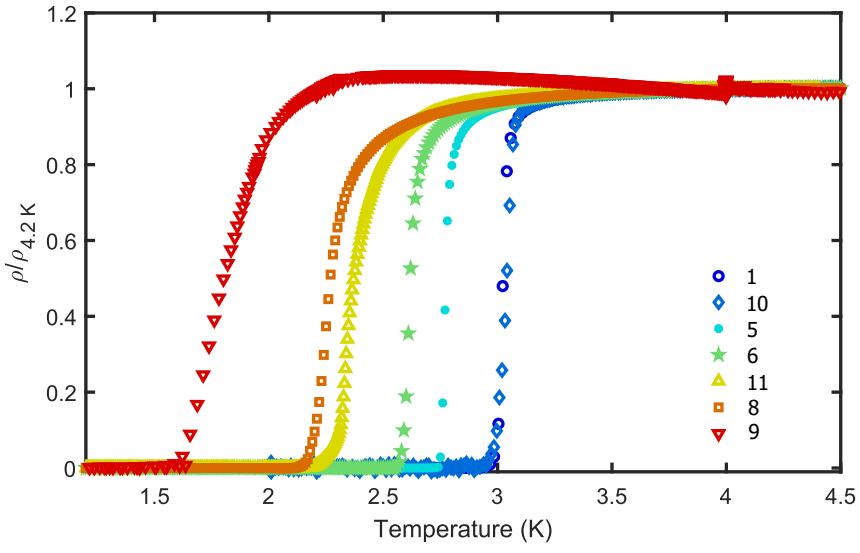

FIG. 1. Temperature dependence of the resistivity, normalized to its value at $4.2 \mathrm{~K}$, showing the SC transition. The legend corresponds to the sample number.

SR830 lock-in amplifier. The current values were in the range of $1 \mu \mathrm{A}$ to $1 \mathrm{nA}$, depending on the sample resistance. Measurements were taken in a commercial Quantum Design PPMS, which enables us to cool down to a temperature of $0.4 \mathrm{~K}$ and apply magnetic fields up to $14 \mathrm{~T}$. Two additional samples with normal-state resistivity of 452 and $3400 \mu \Omega \mathrm{cm}$ were measured in a commercial Oxford Instruments Triton 400 dilution refrigerator from $1 \mathrm{~K}$ to $50 \mathrm{mK}$ and up to $7 \mathrm{~T}$. The normalized resistivity curves for selected samples are shown in Fig. 1. We did not observe the long tails below the transition reported by Dynes [24,25], even for our most resistive samples, possibly because granular aluminum films deposited at low temperature are more homogeneous than those deposited at room temperature since they have a narrower grain-size distribution $[19,20]$. The critical temperature values are in good agreement with previous work on similar films $[16,22,23]$.

Upper critical fields $H_{c 2}(T)$ were obtained by analyzing isotherms of $\rho(H)$, the value of $H_{c 2}(T)$ being defined as that where the resistivity has reached half its normal-state value. Examples of isotherms are shown in the Appendix figures.

Temperature dependences of the critical fields were analyzed in terms of the WHH theory [12], which yields a

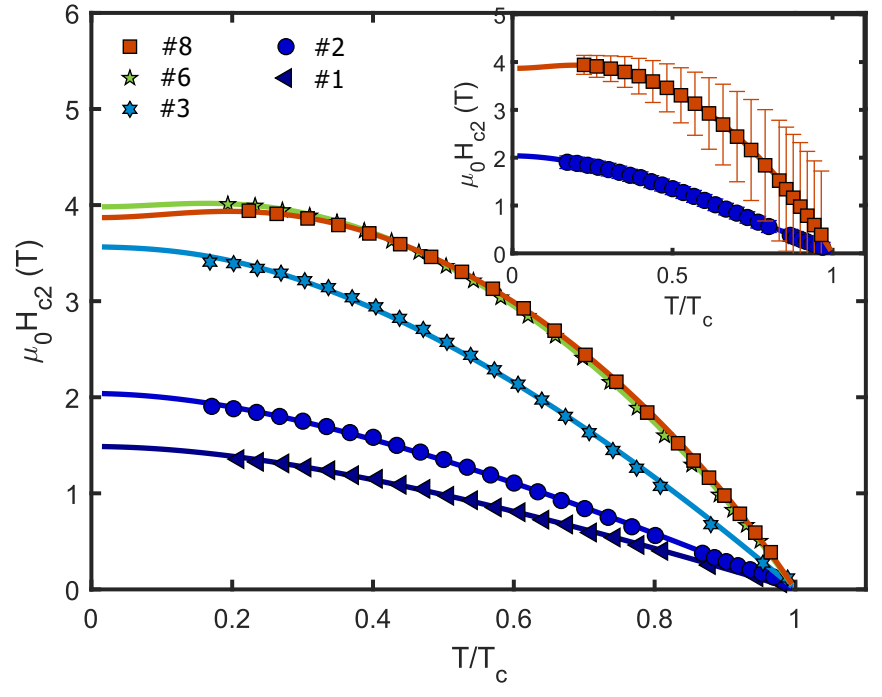

FIG. 2. Temperature dependence of the upper critical field for several of the studied samples. The legend corresponds to the sample number. The data were fitted to Eq. (2). The inset shows part of the data from the main figure, but with bars which mark the value of the field where the resistance has decreased by 90 and 10 percent relatively to the normal-state value.

complete temperature dependence of the upper critical field $H_{c 2}$ of a bulk type-II superconductor with inclusion of Pauli spin paramagnetism and spin-orbit impurity scattering effects. The temperature dependence of $H_{c 2}$ in the dirty limit is given by

$$
\begin{aligned}
\ln \frac{1}{t}= & \sum_{\nu=-\infty}^{\infty}\left\{\frac{1}{|2 v+1|}-\left[|2 v+1|+\frac{\bar{h}}{t}\right.\right. \\
& \left.\left.+\frac{(\alpha \bar{h} / t)^{2}}{|2 v+1|+\left(\bar{h}+\lambda_{s o}\right) / t}\right]^{-1}\right\},
\end{aligned}
$$

where $t=T / T_{c}, \bar{h}=\frac{4}{\pi^{2}} H_{c 2}(T) \frac{1}{\left|d H_{c 2} / d T\right|_{T_{c} T_{c}}}, \alpha$ is the Maki parameter [26], and $\lambda_{s o}=\hbar / 3 \pi k_{B} T_{c} \tau_{s o}$ is the spin-orbit scattering constant. Details of the fitting method, lending values of $\alpha, \Delta$, and $\xi$, are given in the Appendix. Examples of fits are shown in Fig. 2 for selected samples.

TABLE I. Properties of the studied samples. The parameters $\alpha, H_{c 2}$ (the critical field at $T=0$ ), $H_{c 2}^{\text {orb }}$, and $H_{P}$ have been extracted by fitting to Eq. (2).

\begin{tabular}{lcccccc}
\hline \hline ID & $\rho_{4.2 K}^{n}(\mu \Omega \mathrm{cm})$ & $T_{c}(\mathrm{~K})$ & $\alpha$ & $H_{c 2}(\mathrm{~T})$ & $H_{c 2}^{\text {orb }}(\mathrm{T})$ & $H_{P}(\mathrm{~T})$ \\
\hline 1 & 169 & $3.02 \pm 0.05$ & & 1.49 & 1.55 & 14.5 \\
2 & 250 & $3.05 \pm 0.14$ & & 2.04 & 2.10 & 12.5 \\
3 & 547 & $3.05 \pm 0.07$ & $0.69 \pm 0.07$ & 3.57 & 4.34 & $8.97 \pm 0.61$ \\
4 & 973 & $2.99 \pm 0.06$ & $0.85 \pm 0.08$ & 4.13 & 5.43 & $9.06 \pm 0.50$ \\
5 & 1157 & $2.77 \pm 0.03$ & $1.30 \pm 0.03$ & 4.08 & 6.71 & $7.31 \pm 0.05$ \\
6 & 1687 & $2.62 \pm 0.05$ & $1.48 \pm 0.03$ & 3.98 & 7.13 & $6.80 \pm 0.04$ \\
7 & 8300 & $2.33 \pm 0.11$ & $1.76 \pm 0.09$ & 3.91 & 7.92 & $6.38 \pm 0.08$ \\
8 & 8820 & $2.27 \pm 0.12$ & $1.70 \pm 0.03$ & 3.87 & 7.50 & $6.36 \pm 0.03$ \\
9 & 19000 & $1.81 \pm 0.20$ & & $3.83^{\mathrm{a}}$ & & 6.8 \\
10 & 452 & $3.04 \pm 0.04$ & & 3.59 & 3.71 & 6.6 \\
11 & 3400 & $2.37 \pm 0.16$ & $1.43 \pm 0.07$ & 4.19 & 7.32 & $7.26 \pm 0.11$ \\
\hline \hline
\end{tabular}

${ }^{\mathrm{a}} H_{c 2}$ value as measured at $0.4 \mathrm{~K}$. 


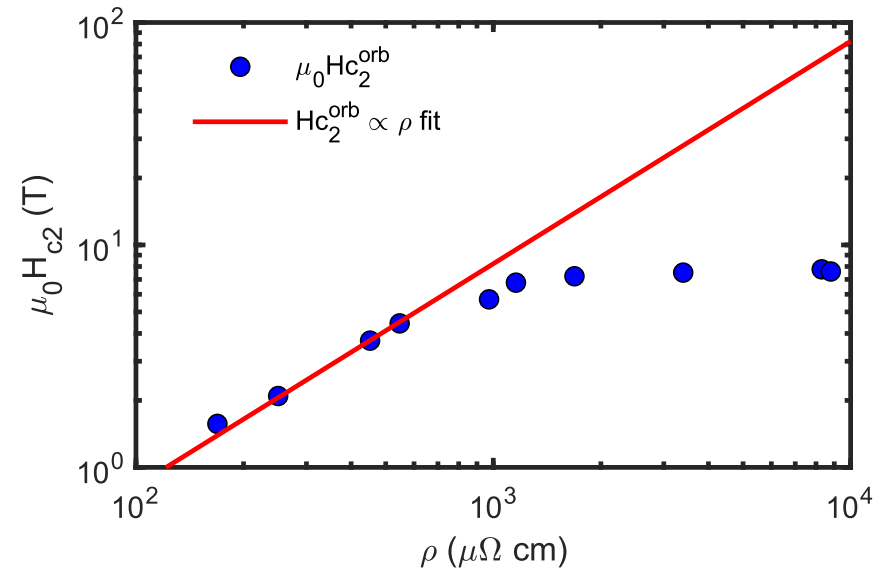

FIG. 3. The orbital critical field $H_{c 2}^{\text {orb }}$ vs resistivity (circles) as obtained from fitting the data to WHH theory along a linear fit to the low-resistivity data.

\section{RESULTS}

The main parameters of the studied samples are summarized in Table I. The Maki parameter increases with resistivity, reaching values higher than unity at resistivities higher than $1000 \mu \Omega \mathrm{cm}$. The error bars deserve a few comments. For $H_{P}$, and therefore for the gap and the strong-coupling ratio, they are large at intermediate resistivity values. It is only for resistivities higher than $1000 \mu \Omega \mathrm{cm}$ that we consider their values to be reasonably accurate. The coherence length saturates down to a value of about 6 to $7 \mathrm{~nm}$. The value of the strong-coupling parameter is in the range of 5 .

For the low-resistivity samples 1,2 , and 10 , the temperature dependence of the critical field is in agreement with theory without taking into account the effect of spin polarization. But for samples having resistivity values higher than several-hundred $\mu \Omega \mathrm{cm}$, one can see from Fig. 2 that it is noticeably different from that of the lower-resistivity samples, particularly at low temperatures. Values of the Maki parameter larger than 1 are necessary to fit the data to the WHH theory for resistivity values larger than $1000 \mu \Omega \mathrm{cm}$. Values of $H_{c 2}^{\text {orb }}$ are plotted in Fig. 3 as a function of the normal-state resistivity. At low resistivities, they increase linearly with $\rho$, as expected for a dirty type-II superconductor. At high resistivities, they saturate at about 7 to $8 \mathrm{~T}$.

We note that for all of the samples, the fit yields $\lambda_{s o}=$ 0 , which deserves a few remarks. The spin-orbit scattering time can be approximated by [27] $\tau_{s o} \simeq \tau /\left(Z \alpha_{f}\right)^{4}$, where $\tau$ is the elastic scattering time, $Z$ is the atomic number, and $\alpha_{f} \simeq 1 / 137$ is the fine-structure constant. Estimating $\tau$ from the relation $\rho \tau=3.13 \times 10^{-14} \mu \Omega \mathrm{cm} \mathrm{s}$ for aluminum [28], we obtain, for the lowest resistivity measured sample, $\tau \simeq 1.9 \times 10^{-16} \mathrm{sec}$ and therefore $\tau_{\text {so }} \simeq \tau \times 1.2 \times 10^{4} \simeq$ $2.3 \times 10^{-12} \mathrm{sec}$. The expected spin-orbit parameter is then about $\lambda_{s o} \sim 0.1$. However, as $\rho$ changes over two orders of magnitude, we expect that so does $\lambda_{\text {so }}$. The fact that we can fit very well the data for all samples without spin-orbit suggests that the actual elastic scattering time $\tau$ is not changing much with increasing resistivity; we will return to this point later on. (a)

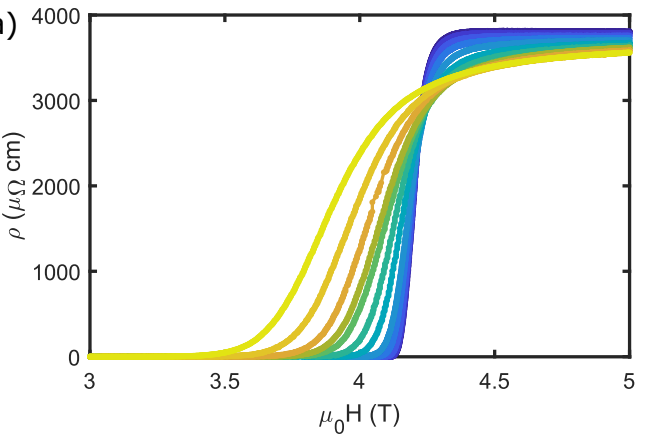

(c)

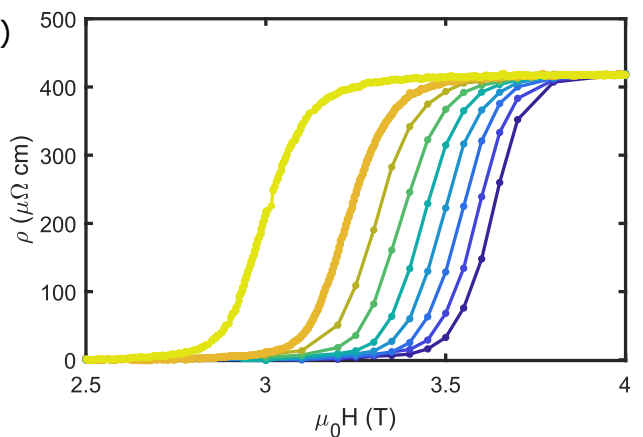

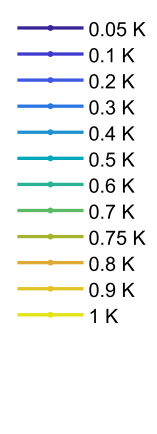

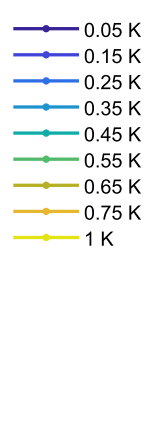

(b)
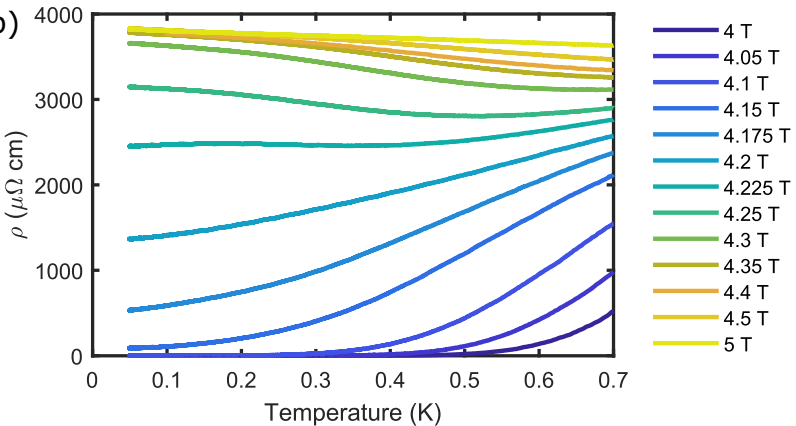

(d)

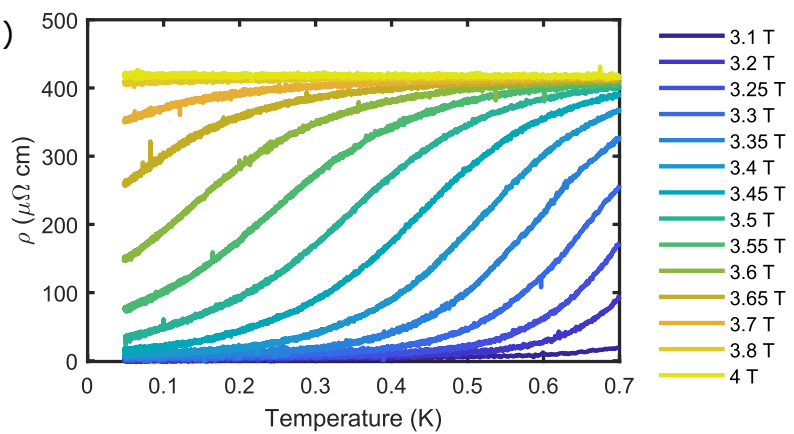

FIG. 4. Resistivity magnetic field and temperature dependence of the samples 10 and 11, measured down to 50 mK. (a),(b) High-resistivity sample with $\rho_{4.2 \mathrm{~K}}=3400 \mu \Omega \mathrm{cm}$. (c),(d) Low-resistivity sample with $\rho_{4.2 \mathrm{~K}}=452 \mu \Omega \mathrm{cm}$. Note that in sharp contrast, the high-resistivity sample transition narrows dramatically as the temperature decreases. Note in (b) that in a narrow field range around $4.25 \mathrm{~T}$, the resistivity goes through a minimum, at a temperature compatible with the occurrence of a maximum in $H_{c 2}(T)$. This is where the transition changes from second to first order. 
For values of the Maki parameter $\alpha$ larger than 1, the WHH theory predicts that the critical field where a second-order transition occurs should actually decrease at low temperatures. A first-order transition is then expected at a higher field. To test these predictions, we have taken some measurements down to $50 \mathrm{mK}$.

Isotherms measured from $1 \mathrm{~K}$ down to $50 \mathrm{mK}$ for a sample with normal-state resistivity of $3400 \mu \Omega \mathrm{cm}$ are shown in Fig. 4(a). Below $0.4 \mathrm{~K}$, the transition becomes extremely sharp. The long tails seen at higher temperatures above the critical field are suppressed. The same behavior was reported by Tedrow and Meservey [7] on thin Al films in a parallel field configuration, which they attributed to a change from a second-order transition to a first-order one, as predicted by Fulde and Maki [29]. This change is accompanied by a suppression of the paraconductivity above the critical field, which for a three-dimensional sample in perpendicular field [29] is of the form $\sigma_{f l} \propto T / \sqrt{H-H_{c 2}}$. The resistivity was fitted to the expression $\rho=\frac{1}{1 / \rho_{n}+a T / \sqrt{H-H_{C 2}(T)}}$, where $\rho_{n}$ was chosen here as the value above the critical field at $50 \mathrm{mK}$ and the fitting parameters are $a$ and $H_{c 2}$. The fitting range was limited down to a field value at which $\rho=0.85 \rho_{n}$. The extracted $H_{c 2}(T)$ values from the fit are shown in Fig. 5(a), where one can see the expected downturn of $H_{c 2}(T)$ (below $\sim 0.7 \mathrm{~K}$ ) as predicted by WHH for $\alpha>1$, where a first-order transition is expected. Deviation from a linear dependence on $T$ can be seen in Fig. 5(b), at magnetic fields close to $H_{c 2}(T)$, where the paraconductivity becomes more sensitive to the anomalous $H_{c 2}$ temperature dependence. This is in sharp contrast to the excellent agreement at higher magnetic field values up to $5 \mathrm{~T}$, where the temperature dependence of $H_{c 2}$ is negligible.

The same similarity is seen between their cooling curves and our own shown in Fig. 4(b), displaying a striking minimum at $4.25 \mathrm{~T}$. By contrast, isotherms measured in the same range of temperature for a more metallic sample with normal-state resistivity of $452 \mu \Omega \mathrm{cm}$ show a regular shift of the transition down to the lowest temperature, as can be seen in Figs. 4(c) and 4(d). We conclude that granular films near the metal-insulator transition do undergo a first-order field-induced transition, in keeping with the large values of the Maki parameter that we have determined.

\section{DISCUSSION AND CONCLUSIONS}

While, according to Eq. (1), the value of the Maki parameter can, in principle, be increased beyond 1 by reducing the transport scattering time, strong scattering generally leads to Anderson electron localization and destruction of superconductivity before this regime is reached. This is well documented for $\mathrm{NbN}_{x}$, where scattering is increased by introducing nitrogen vacancies [30,31]. To discuss this point quantitatively, it is useful to rewrite Eq. (1) as

$$
\alpha=\frac{3}{2 k_{F} l},
$$

where we have assumed that the effective mass of the electron is that of a free electron. Reaching values of $\alpha$ of the order of unity requires that the product $k_{F} l$ approaches unity. But in $\mathrm{NbN}_{x}$ [30-32] and other similar atomically disordered mate-
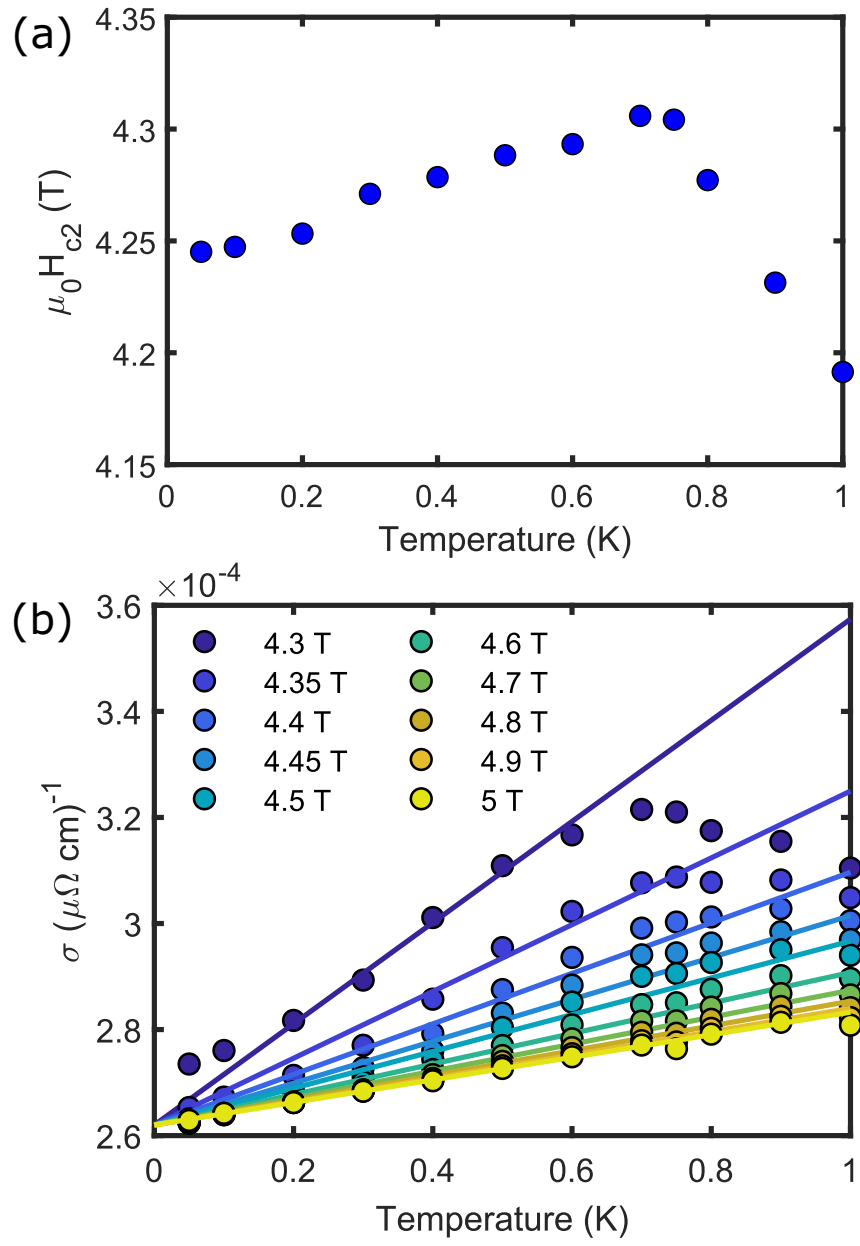

FIG. 5. Sample 11 paraconductivity analysis. (a) $H_{c 2}(T)$ as extracted from fitting the $\rho(H)$ data to Fulde and Maki theory [29], as described in the text. (b) Conductivity $\sigma$ vs temperature at different values of magnetic field. Circles are the data as extracted from the $\rho(H)$ curves and the thick lines are linear fits to the Fulde and Maki theory, $\sigma=\sigma_{n}+a T / \sqrt{H-H_{c 2}(T)}$. $\sigma_{n}$ was chosen as the intercept at the highest field measured $(5 \mathrm{~T})$ and was fixed for all fits.

rials such as MoN [33], superconductivity is then completely quenched. Therefore, Pauli limitation cannot be observed in that case.

But when a transition of the Mott type is approached, superconductivity is not so quickly destroyed because the density of states at the Fermi level remains unchanged [15,34]. The Fermi energy is reduced and the effective electronic mass is strongly enhanced [15]. The value of the Maki parameter must then take into account this enhancement factor. Even if $k_{F} l$ is smaller than unity, as is the case in granular aluminum [16], superconductivity persists and the Maki parameter can be larger than 1 . The large value of the Maki parameter that we have found near the metal-to-insulator transition of granular aluminum is therefore an important confirmation that in this material, the transition is of the Mott type rather than of the Anderson type.

In fact, near the transition, the increase of the Maki parameter is driven by the renormalization of the Fermi energy rather 

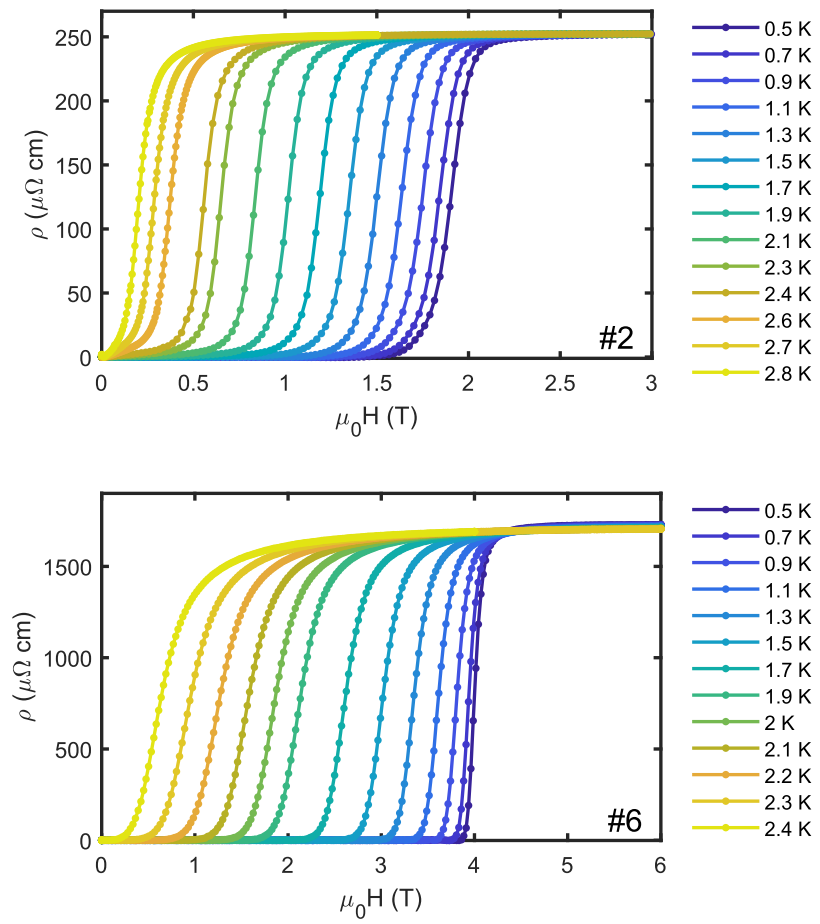
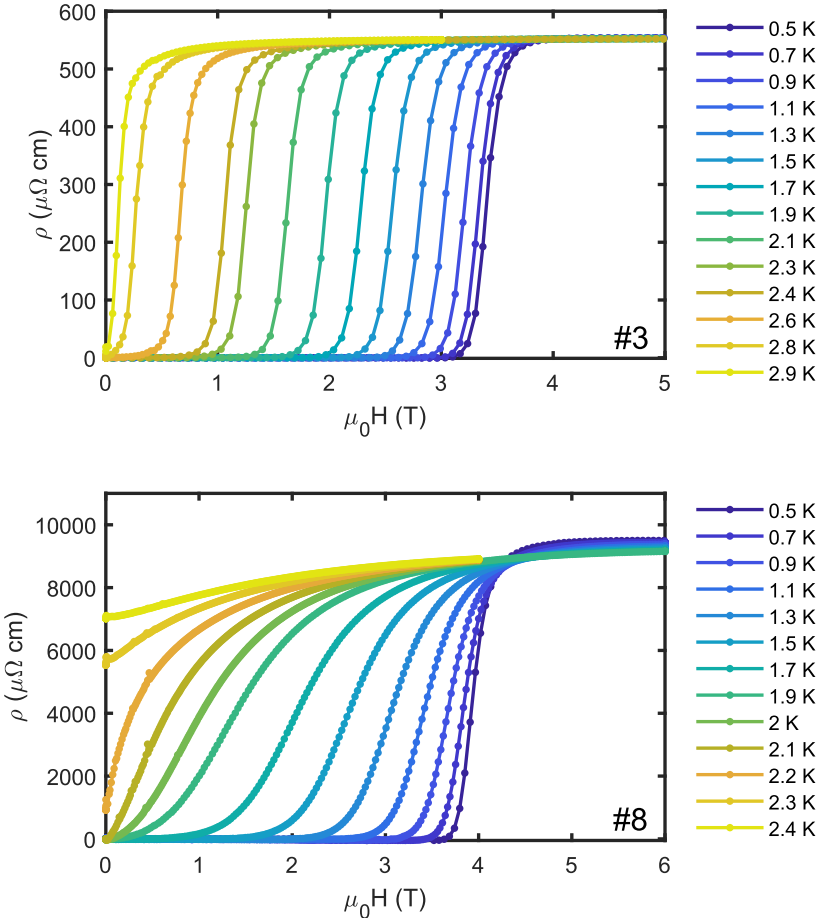

FIG. 6. Magnetic field dependence of the resistivity, showing $\rho(H)$ isotherms of four samples. Note that for the high-resistivity samples 6 and 8 , the transition becomes narrow as the temperature decreases.

than by the decrease of the scattering time, which dominates far from the transition.

Since at the Mott transition the effective Fermi energy goes to zero, it becomes at some point of the same order of magnitude as the energy gap. Here, strong coupling is the result of the reduced Fermi energy. When it becomes of the same order as the gap, the number of Cooper pairs per coherence volume is of the order of unity. The transition region can be treated as a BCS-BEC crossover region. Theory predicts that in this region, the phase-coherence length, and hence the orbital critical field, saturates [35]. This is in agreement with the behavior that we observe. The value of about 5 that we have obtained for the strong-coupling parameter is also in fair agreement with that predicted in the BCS-BEC crossover region $[16,36,37]$.

In conclusion, by studying the temperature dependence of the upper critical field of granular aluminum films and fitting it to the WHH theory, we have been able to determine the values of the Maki parameter, the Pauli field, and the phasecoherence length near the metal-to-insulator transition. The value reached by the Maki parameter is larger than one. This requires a strong mass renormalization factor. For the highestresistivity films, the transition becomes first order below a few $100 \mathrm{mK}$. These results are consistent with a Mott transition, not with an Anderson transition. The strong-coupling ratio derived from the values of the Pauli field and of the measured critical temperature reaches a value of about 5 , while the phase-coherence length saturates to a value of about 6 to $7 \mathrm{~nm}$. These findings are consistent with a BCS-BEC crossover regime.
Note added in proof. Recently, we became aware of the possible relevance of a first-order transition to a recent work [38] showing that the performance of a granular aluminum quantum device remains unaffected by magnetic fields of up to 1 Tesla. We are grateful to Ioan Pop for making us aware of this.

\section{ACKNOWLEDGMENTS}

We thank G. Strinati for stimulating discussions, Y. Dagan for letting us take measurements in his dilution refrigerator, and G. Tuvia for his assistance with measurements taken in the dilution refrigerator.

\section{APPENDIX: DATA ANALYSIS OF $\rho(H)$ AND ADDITIONAL DATA}

The fitting of the critical field data was done by first constructing a $H_{c 2}(t)$ curve from Eq. (2) in the main text. This was done by solving it numerically for $H_{c 2}(t)$ at each value of $t=T / T_{c}, \alpha$, and $\left|d H_{c 2} / d T\right|_{T_{c}}$. The reason we chose the slope near $T_{c}$ as a fitting parameter is a lack of accurate data points near $T_{c}$ for most of the samples. Utilizing MATLAB, the values of $\alpha$ and $\left|d H_{c 2} / d T\right|_{T_{c}}$ were varied until the best fit was obtained, where the uncertainty of both fitting parameters was determined by a $95 \%$ confidence interval. Upon finding the best values, $H_{c 2}(0)$ is found by evaluating Eq. (2) in the main text at $t \rightarrow 0$. Then, $H_{c 2}^{\text {orb }}$ and $H_{P}$ can be retrieved by

$$
H_{c 2}^{\mathrm{orb}}=\sqrt{1+\alpha^{2}} H_{c 2}(0),
$$




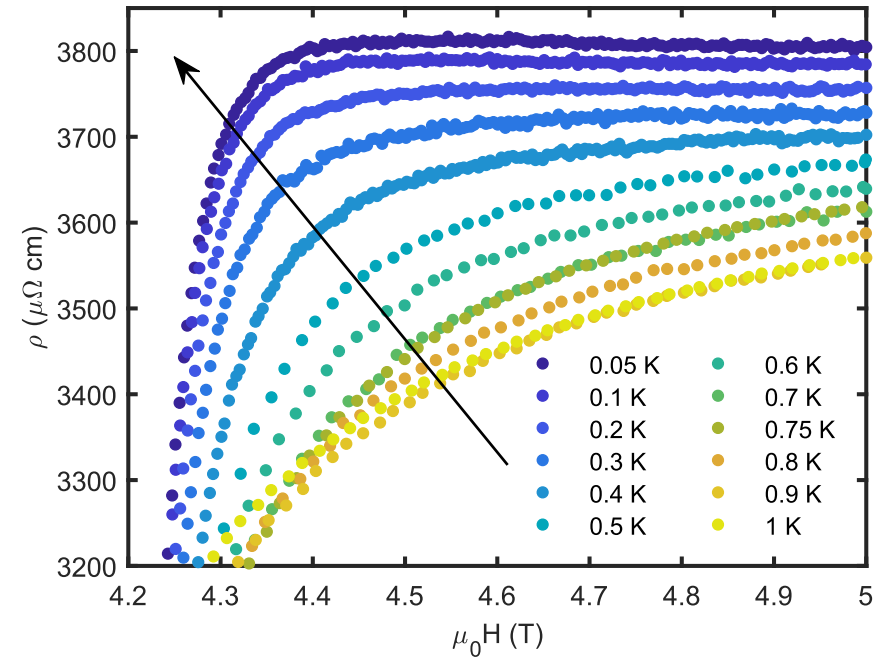

FIG. 7. $\rho(H)$ isotherms of sample 11, zoomed into the regime $H>H_{c 2}$. The arrow marks the direction of decreasing temperature.

$$
H_{P}(0)=\frac{\sqrt{2}}{\alpha} H_{c 2}^{\mathrm{orb}}(0)
$$

The Pauli field gives access to the gap parameter

$$
H_{P}=\frac{\Delta}{g^{1 / 2} \mu_{B}}
$$

and the orbital field to the coherence length

$$
H_{c 2}^{\mathrm{orb}}=\frac{\Phi_{0}}{2 \pi \xi^{2}} .
$$

We have thus obtained the values of $\alpha, \Delta$, and $\xi$.

Isotherms of selected samples are shown in Fig. 6 . Isotherms of sample 11, measured down to $50 \mathrm{mK}$, are shown in Fig. 7.
[1] T. Coffey, C. Martin, C. C. Agosta, T. Kinoshota, and M. Tokumoto, Bulk two-dimensional Pauli-limited superconductor, Phys. Rev. B 82, 212502 (2010).

[2] C. C. Agosta, Jing Jin, W. A. Coniglio, B. E. Smith, K. Cho, I. Stroe, C. Martin, S. W. Tozer, T. P. Murphy, E. C. Palm, J. A. Schlueter, and M. Kurmoo, Experimental and semiempirical method to determine the Pauli-limiting field in quasi-two-dimensional superconductors as applied to $\kappa$ $(\mathrm{BEDT}-\mathrm{TTF})_{2} \mathrm{Cu}(\mathrm{NCS})_{2}$ : Strong evidence of a FFLO state, Phys. Rev. B 85, 214514 (2012).

[3] T. Suzuki, Y. Seguchi, and T. Tsuboi, Fermi liquid effect on tricritical superconducting transitions in thin tin films under the spin paramagnetic limitation, J. Phys. Soc. Jpn. 69, 1462 (2000).

[4] P. W. Adams, P. Herron, and E. I. Meletis, First-order spinparamagnetic transition and tricritical point in ultrathin $\mathrm{Be}$ films, Phys. Rev. B 58, R2952(R) (1998).

[5] X. S. Wu, P. W. Adams, and G. Catelani, Density of states, entropy, and the superconducting Pomeranchuk effect in Paulilimited Al films, Phys. Rev. B 74, 144519 (2006).

[6] V. Yu. Butko, P. W. Adams, and I. L. Aleiner, Exchange Fields and the Finite Bias Tunneling Anomaly in Paramagnetically Limited Superconducting Al Films, Phys. Rev. Lett. 82, 4284 (1999).

[7] P. M. Tedrow and R. Meservey, Spin-paramagnetic effects in superconducting aluminum films, Phys. Rev. B 8, 5098 (1973).

[8] S. Khim, B. Lee, J. W. Kim, E. S. Choi, G. R. Stewart, and K. H. Kim, Pauli-limiting effects in the upper critical fields of a clean life as single crystal, Phys. Rev. B 84, 104502 (2011).

[9] Z. Wei, Ge He, W. Hu, Z. Feng, X. Wei, C. Y. Ho, Q. Li, J. Yuan, C. Xi, Z. Wang, Q. Chen, B. Zhu, F. Zhou, X. Dong, Li Pi, A. Kusmartseva, F. V. Kusmartsev, Z. Zhao, and K. Jin, Anomalies of upper critical field in the spinel superconductor $\mathrm{LiTi}_{2} \mathrm{O}_{4-\delta}$, Phys. Rev. B 100, 184509 (2019).

[10] T. Chui, P. Lindenfeld, W. L. McLean, and K. Mui, Coupling and isolation: Critical field and transition temperature of superconducting granular aluminum, Phys. Rev. B 24, 6728 (1981).
[11] S. A. Dodds, S. N. Harrington, R. S. Newrock, and K. Loeffler, Evidence for large-scale structures in granular superconductors, Phys. Rev. B 33, 3115 (1986).

[12] N. R. Werthamer, E. Helfand, and P. C. Hohenberg, Temperature and purity dependence of the superconducting critical field, $H_{c 2}$. III. Electron spin and spin-orbit effects, Phys. Rev. 147, 295 (1966).

[13] P. Fulde and R. A. Ferrell, Superconductivity in a strong spinexchange field, Phys. Rev. 135, A550 (1964).

[14] A. I. Larkin and Y. N. Ovchinnikov, Nonuniform state of superconductors, Zh. Eksp. Teor. Fiz. 47, 1136 (1964) [Sov. Phys. JETP 20, 762 (1965)].

[15] A. Georges, G. Kotliar, W. Krauth, and M. J. Rozenberg, Dynamical mean-field theory of strongly correlated fermion systems and the limit of infinite dimensions, Rev. Mod. Phys. 68, 13 (1996).

[16] A. G. Moshe, E. Farber, and G. Deutscher, Optical conductivity of granular aluminum films near the Mott metal-to-insulator transition, Phys. Rev. B 99, 224503 (2019).

[17] U. S. Pracht, N. Bachar, L. Benfatto, G. Deutscher, E. Farber, M. Dressel, and M. Scheffler, Enhanced Cooper pairing versus suppressed phase coherence shaping the superconducting dome in coupled aluminum nanograins, Phys. Rev. B 93, 100503(R) (2016).

[18] N. Bachar, U. Pracht, E. Farber, M. Dressel, G. Deutscher, and M. Scheffler, Signatures of unconventional superconductivity in granular aluminum, J. Low Temp. Phys. 179, 83 (2015).

[19] G. Deutscher, M. Gershenson, E. Grunbaum, and Y. Imry, Granular superconducting films, J. Vac. Sci. Technol. 10, 697 (1973).

[20] S. Lerer, N. Bachar, G. Deutscher, and Y. Dagan, Nernst effect beyond the coherence critical field of a nanoscale granular superconductor, Phys. Rev. B 90, 214521 (2014).

[21] A. Moshe, N. Bachar, S. Lerer, Y. Lereah, and G. Deutscher, Multi-level Kondo effect and enhanced critical temperature in nanoscale granular Al, J. Supercond. Novel Magn. 31, 733 (2017). 
[22] N. Bachar, S. Lerer, S. Hacohen-Gourgy, B. Almog, and G. Deutscher, Kondo-like behavior near the metal-to-insulator transition of nanoscale granular aluminum, Phys. Rev. B 87, 214512 (2013).

[23] N. Bachar, S. Lerer, A. Levy, S. Hacohen-Gourgy, B. Almog, H. Saadaoui, Z. Salman, E. Morenzoni, and G. Deutscher, Mott transition in granular aluminum, Phys. Rev. B 91, 041123(R) (2015).

[24] R. C. Dynes and J. P. Garno, Metal-Insulator Transition in Granular Aluminum, Phys. Rev. Lett. 46, 137 (1981).

[25] R. C. Dynes, J. P. Garno, G. B. Hertel, and T. P. Orlando, Tunneling Study of Superconductivity Near the Metal-Insulator Transition, Phys. Rev. Lett. 53, 2437 (1984).

[26] K. Maki, Effect of pauli paramagnetism on magnetic properties of high-field superconductors, Phys. Rev. 148, 362 (1966).

[27] A. A. Abrikosov, Spin-orbit interaction and the Knight shift in superconductors, Sov. Phys. JETP 15, 752 (1962).

[28] D. Gall, Electron mean free path in elemental metals, J. Appl. Phys. 119, 085101 (2016).

[29] P. Fulde and K. Maki, Fluctuations in high field superconductors, Z. Phys. A: Hadrons Nucl. 238, 233 (1970).

[30] M. Mondal, A. Kamlapure, M. Chand, G. Saraswat, S. Kumar, J. Jesudasan, L. Benfatto, V. Tripathi, and P. Raychaudhuri, Phase Fluctuations in a Strongly Disordered $s$-Wave NbN Superconductor Close to the Metal-Insulator Transition, Phys. Rev. Lett. 106, 047001 (2011).

[31] B. Cheng, L. Wu, N. J. Laurita, H. Singh, M. Chand, P. Raychaudhuri, and N. P. Armitage, Anomalous gap-edge dissipation in disordered superconductors on the brink of localization, Phys. Rev. B 93, 180511(R) (2016).

[32] M. Mondal, M. Chand, A. Kamlapure, J. Jesudasan, V. C. Bagwe, S. Kumar, G. Saraswat, V. Tripathi, and P. Raychaudhuri, Phase diagram and upper critical field of homogeneously disordered epitaxial 3-dimensional $\mathrm{NbN}$ films, J. Supercond. Novel Magn. 24, 341 (2011).

[33] K. Makise, F. Ichikawa, T. Asano, and B. Shinozaki, Fieldtuned superconductor-insulator transitions and hall resistance in thin polycrystalline MoN films, J. Phys.: Condens. Matter 30, 065402 (2018).

[34] M. Capone, M. Fabrizio, C. Castellani, and E. Tosatti, Strongly correlated superconductivity, Science 296, 2364 (2002).

[35] A. Spuntarelli, P. Pieri, and G. C. Strinati, Solution of the Bogoliubov-de Gennes equations at zero temperature throughout the BCS-BEC crossover: Josephson and related effects, Phys. Rep. 488, 111 (2010).

[36] L. Pisani, P. Pieri, and G. C. Strinati, Gap equation with pairing correlations beyond the mean-field approximation and its equivalence to a Hugenholtz-Pines condition for fermion pairs, Phys. Rev. B 98, 104507 (2018).

[37] L. Pisani, A. Perali, P. Pieri, and G. C. Strinati, Entanglement between pairing and screening in the Gorkov-MelikBarkhudarov correction to the critical temperature throughout the BCS-BEC crossover, Phys. Rev. B 97, 014528 (2018).

[38] K. Borisov, D. Rieger, P. Winkel, F. Henriques, F. Valenti, A. Ionita, M. Wessbecher, M. Spiecker, D. Gusenkova, I. M. Pop, and W. Wernsdorfer, Superconducting granular aluminum resonators resilient to magnetic fields up to 1 Tesla, Appl. Phys. Lett. 117, 120502 (2020). 